\title{
Therapeutic Intranodal Lymphangiography for Refractory Chylous Ascites Complicating Acute Necrotic Pancreatitis
}

\author{
Thomas Jardinet ${ }^{1}$, Len Verbeke ${ }^{2}$, Lawrence Bonne ${ }^{1}$, Geert Maleux ${ }^{1}$
}

1) Department of Radiology,

University Hospitals Leuven,

Leuven

2) Department of

Gastroenterology and

Hepatology, University

Hospitals Leuven, Leuven

Belgium

\author{
Address for correspondence: \\ Geert Maleux, MD, $\mathrm{PhD}$ \\ Department of Radiology, \\ University Hospitals Leuven \\ Herestraat 49, \\ B-3000 Leuven \\ Belgium \\ geert.maleux@uzleuven.be
}

Received: 21.03.2018

Accepted: 31.05 .2018

\begin{abstract}
Chylous ascites is a rare complication of acute pancreatitis. However, the incidence of intraperitoneal chyle leakage related to severe pancreatitis may be much higher. This is probably the result of direct damage to the cisterna chyli or its tributaries by pancreatic enzymes. In this case, conservative treatment failed to resolve the chyle leak. For the first time, to our knowledge, ultrasound guided therapeutic intranodal lymphangiography was shown to be a successful, minimally invasive treatment option in chylous ascites complicating acute necrotic pancreatitis.
\end{abstract}

Key words: chylous ascites - pancreatitis - lymphangiography.

Abbreviations LCT: long chain triglycerides; MCT: medium chain triglycerides; TPN: total parenteral nutrition; WON: walled-off necrosis.

\section{INTRODUCTION}

The lymphatic system of the soft tissues, the intestinal lymphatic system and the hepatic lymphatic system all communicate with each other and eventually drain into the cisterna chyli and the thoracic duct [1]. Besides drainage of excessive interstitial fluid, the main function of the intestinal lymphatics is the transportation of dietary fats. Intestinal chyle contains high concentrations of converted long chain triglycerides (LCT). Intraperitoneal chylous leakage can present with painless abdominal distension, but it can also lead to significant fluid loss, protein loss, severe malnutrition and susceptibility to infection [2].

Chylous ascites was previously considered to be a rare complication of acute pancreatitis, with only a few case reports found in the literature
[3-11], until a Chinese group found that chylous ascites was present in $15 \%$ of severe cases of acute pancreatitis [12]. The intraperitoneal chyle leak is probably caused by direct damage to the cisterna chyli or its tributaries by pancreatic enzymes. Another possible pathogenesis is obstruction of the major lymph vessels causing leakage of chyle out of dilated small subserosal lymph vessels.

The initial treatment is usually conservative, consisting of total parenteral nutrition (TPN) or a diet rich in medium chain triglycerides (MCT), as these types of triglycerides are directly absorbed into the portal circulation, lowering the flow in the intestinal lymphatic system [3].

\section{CASE REPORT}

A 71-year-old man was referred for complications of acute necrotizing biliary pancreatitis, with two months earlier onset. The patient presented with walled-off necrosis (WON), intrapancreatic common bile duct obstruction and splenic vein thrombosis. During the following four months he underwent repeated endoscopic bile duct stenting and multiple endoscopic ultrasound-guided transgastric pancreatic necrosectomy procedures because of infected WON. The patient was unsuitable for pancreatic surgery due to the splenic vein thrombosis and the subsequent formation of collateral vessels. A gastrostomy tube was also put in place to allow enteral feeding given his poor nutritional status. The cholestasis resolved and the volume of the WON diminished. However, 
the patient developed intractable ascites necessitating weekly therapeutic paracenteses in the outpatient clinic. The fluid was milky with increased triglyceride level (varying between 281 and $369 \mathrm{mg} / \mathrm{dL}$ ) and low amylase content $(6 \mathrm{U} / \mathrm{L})$, consistent with chylous ascites. Other causes of post-pancreatitis ascites, including exocrine pancreatic insufficiency, were excluded. A diet consisting only of low fat and high protein enteral nutrition enriched with MCT was started, along with a monthly subcutaneous administration of lanreotide (90 mg). Following this, only a slight and temporary reduction in the paracentesis frequency was noted.

One month after the last endoscopic necrosectomy procedure, the patient presented again at the emergency department because of a worsened general condition with anorexia, severe weight loss and muscle wasting. Computed tomography (CT) revealed an increased volume of ascites, and a WON with volume unchanged (Fig. 1).

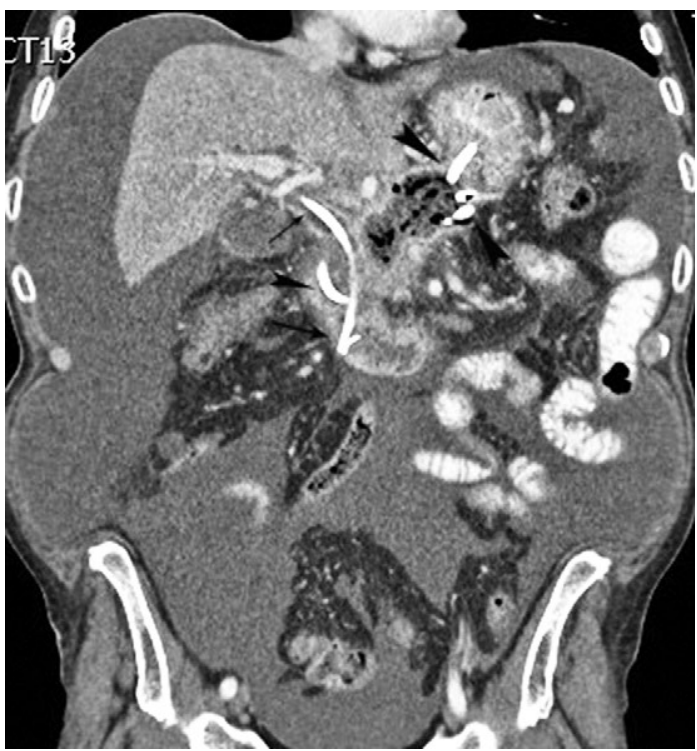

Fig. 1. Coronal abdominal CT scan showing the refractory intraperitoneal chylous fluid. Note also the plastic biliary stent in situ (small arrows) and the gastrocystostomy drain (arrowheads).

Due to the failure of conservative treatment and development of refractory chylous ascites with severe impact on the patient's quality of life, overall fitness and nutritional status, the decision was made to perform a therapeutic intranodal lymphangiography. After bilateral ultrasoundguided puncture of inguinal lymph nodes with a $22 \mathrm{G}$ spinal needle, ethiodized oil (Lipiodol Ultrafluid ${ }^{\circledR}$, Guerbet, Aulnaysous-Bois, France) was slowly injected. The progression of the ethiodized oil through the iliac lymph nodes, the lumbar lymphatic ducts, the cisterna chyli and the thoracic duct was observed under fluoroscopic guidance (Fig. 2). A total of $30 \mathrm{cc}$ ethiodized oil was injected. No contrast extravasation was observed. The patient required two more paracentesis procedures within the first week, after which the ascites resolved and no other intervention was required during followup of 12 months. Shortly after, nutrition support and lanreotide were also stopped, a normal diet was re-introduced and the patient's overall condition improved significantly, with a full recovery of body weight, general condition, muscle mass and performance status.

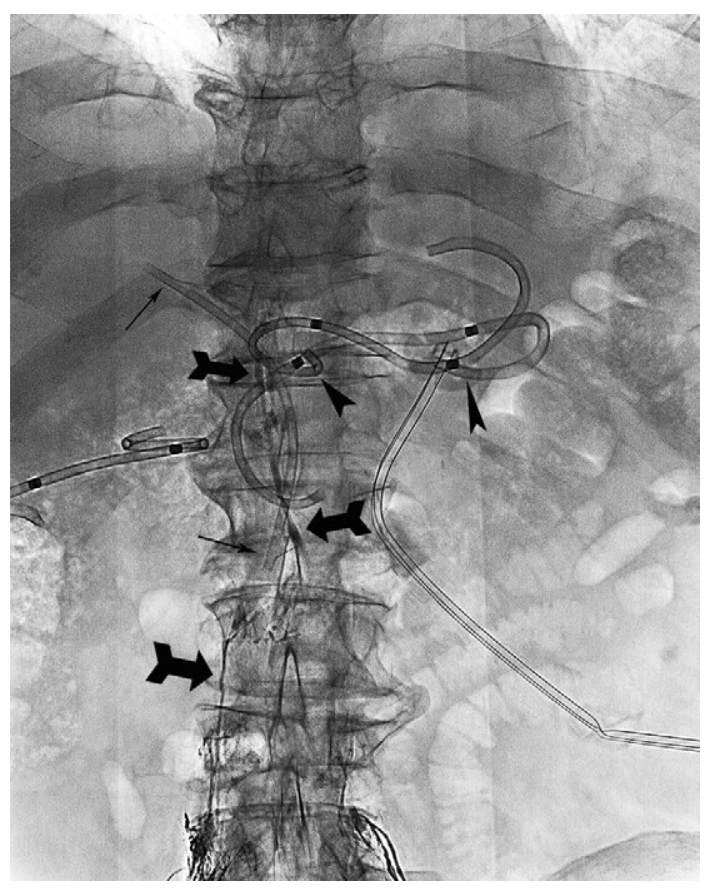

Fig. 2: Fluoroscopic image of the abdomen showing the progression of ethiodized oil through the inguinal and iliac lymphatic channels with opacification of the retroperitoneal lymph vessels and cisterna chyli (bold arrows). Note also the plastic biliary stent (small arrows) and gastrocystostomy (arrowheads) in situ.

\section{DISCUSSION}

The treatment of chylous ascites is usually conservative, consisting of TPN or a MCT-rich diet, lowering the flow in the intestinal lymphatic system [3]. Early enteral nutrition, on the other hand, increases the risk of chylous ascites $[12,13]$. If conservative management fails, bipedal [13] or intranodal [2] lymphangiography should be attempted. Potential complications related to the intralymphatic injection of ethiodized oil are rare and include paradoxical cerebral embolization and lung emboli [14]. Other treatment options are somatostatin analogues, repeat paracentesis or insertion of a tunneled peritoneal drainage catheter [15].

Intranodal lymphangiography with use of Lipiodol ${ }^{\circledast}$ has never previously been reported as a therapeutic option in post-pancreatitis chylous ascites, although it has been used as a treatment in other causes of refractory chyle leakage, such as pancreatic surgery, as reported in the study of Tabchouri et al. [13]. In this retrospective analysis dealing with postoperative chylous ascites following pancreatic surgery, 5 out of 15 patients required additional bipedal Lipiodol-based lymphangiography after failed conservative management; no risk factors for failure of conservative management were identified [13]. In a larger study, including patients with both refractory chylothorax and chylous ascites, lymphangiography led to complete occlusion of lymph leaks of various origins in $70.3 \%$ of cases $(96.8 \%$ of 
patients with a drainage volume of less than $200 \mathrm{~mL} /$ day); no recurrence was observed, and no major complications were described [16]. The ethiodized oil is believed to accumulate at the leakage point, leading to inflammatory changes acting as an embolic agent [17].

\section{CONCLUSION}

We report here a case of successful treatment of refractory chylous ascites associated with acute necrotizing pancreatitis. To the best of our knowledge, this is the first time that ultrasound guided therapeutic intranodal lymphangiography with ethiodized was shown to be a successful, minimally invasive treatment option in a case of chylous ascites complicating acute necrotic pancreatitis.

Coflicts of interest: None to declare.

Authors' contribution: T.J. and G.M. collected the data; T.J., L.V., L.B. and G.M. analysed and interpreted the data and wrote the article. All authors revised the manuscript and approved the final version.

\section{REFERENCES}

1. Hsu MC, Itkin M. Lymphatic anatomy. Tech Vasc Interv Radiol 2016;19:247-254. doi:10.1053/j.tvir.2016.10.003

2. Tamura T, Kubo N, Yamamoto A, et al. Cervical chylous leakage following esophagectomy that was successfully treated by intranodal lipiodol lymphangiography: a case report. BMC Surg 2017;17:20. doi:10.1186/s12893-017-0218-x

3. Al-Ghamdi MY, Bedi A, Reddy SB, Tanton RT, Peltekian KM. Chylous ascites secondary to pancreatitis: management of an uncommon entity using parenteral nutrition and octreotide. Dig Dis Sci 2007;52:22612264. doi:10.1007/s10620-006-9734-8

4. Gomez-Martin JM, Martinez-Molina E, Sanjuanbenito A et al. Chylous ascites secondary to acute pancreatitis: a case report and review of the literature. Nutr Hosp 2012;27:314-318.

5. Baban CK, Murphy M, O'Sulleabháin C, O’Hanlon D. Chylous ascites as a consequence of idiopathic pancreatitis. BMJ Case Rep 2014. doi:10.1136/bcr-2013-200132
6. Lippey JF, Yong TL. Chyle leak following biliary pancreatitis. J Surg Case Rep 2014;2014. doi:10.1093/jscr/rju052

7. Yang AL, Baron RH, Hart PA. An uncommon cause of peripancreatic fluid. Gastroenterology 2015;149:e3-4. doi:10.1053/j.gastro.2015.02.022

8. Kumar A, Mandavdhare HS, Rana SS, Gupta R, Sharma V. Chylous ascites due to idiopathic chronic pancreatitis managed with endoscopic stenting. Clin Res Hepatol Gastroenterol 2018;42:e29-e31. doi:10.1016/j. clinre.2017.05.009

9. Poo S, Pencavel TD, Jackson J, Jiao LR. Portal hypertension and chylous ascites complicating acute pancreatitis: the therapeutic value of portal vein stenting. Ann R Coll Surg Engl 2018;100:e1-e3. doi:10.1308/ rcsann.2017.0078

10. Santos MA, Bose PS, Maher S, Desai M. Chylous ascites: an unusual complication of necrotizing pancreatitis. Am J Med 2017;130:e151-e152. doi:10.1016/j.amjmed.2016.11.034

11. D’Amata G, Rega M, Viola V, Bove V, Simeone P, Baiano G. Chyloperitoneum associated with idiopathic pancreatitis: case report and review of the literature. G Chir 2016;37:167-170. doi:10.11138/ gchir/2016.37.4.167

12. Zhang SY, Liang ZY, Yu WQ, Wang ZE, Chen ZB, Zhang Y. Early enteral nutrition with polymeric feeds was associated with chylous ascites in patients with severe acute pancreatitis. Pancreas 2014;43:553-558. doi:10.1097/MPA.0000000000000067

13. Tabchouri N, Frampas E, Marques F, Blanchard C, Jirka A, Regenet N. Chylous ascites management after pancreatic surgery. World J Surg. 2017;41:1054-1060. doi:10.1007/s00268-016-3772-y

14. Sheybani A, Gaba R, Minocha J. Cerebral embolization of ethiodized oil following intranodal lymphangiography. Semin Intervent Radiol 2015;32:10-13. doi:10.1055/s-0034-1396957

15. Maleux G, Indesteege I, Laenen A, Verslype C, Vergote I, Prenen H Tenckhoff tunneled peritoneal catheter placement in the palliative treatment of malignant ascites: technical results and overall clinical outcome. Radiol Oncol 2016;50:197-203. doi:10.1515/raon-20160002

16. Gruber-Rouh T, Naguib NN, Lehnert T, et al. Direct lymphangiography as treatment option of lymphatic leakage: indications, outcomes and role in patient's management. Eur J Radiol 2014;83:2167-2171. doi:10.1016/j. ejrad.2014.09.013

17. Matsumoto T, Yamagami T, Kato T, et al. The effectiveness of lymphangiography as a treatment method for various chyle leakages. Br J Radiol 2009;82:286-290. doi:10.1259/bjr/64849421 